\title{
THE PLANE IS NOT COMPACTLY GENERATED BY A FREE MAPPING
}

\author{
BY \\ S. A. ANDREA
}

\begin{abstract}
Let $X$ denote the plane, or the closed half-plane, and let $T: X \rightarrow X$ be a self homeomorphism which preserves orientation and has no fixed points. It is proved that, if $A$ is any compact subset of $X$, then there exists an unbounded connected subset $B$ of $X$ which does not meet $T^{n}(A)$ for any integer $n$.
\end{abstract}

0. Introduction. The aim of this paper is to furnish a proof of the following result.

THEOREM. Let $X$ be the plane, or a closed half-plane.

Let $T: X \rightarrow X$ be a homeomorphism which preserves orientation and has no fixed points. Then for any compact set $A \subset X$ there exists an unbounded connected set $B \subset X$ which avoids $\bigcup_{-\infty}^{+\infty} T^{n} A$.

This theorem was used by the author in [3] and [4], where flows and topological translations of the plane were studied.

For the duration of the paper we consider only the case $X=\boldsymbol{R}^{2}$. The extension of the theorem to half-planes will be deduced in the final section.

The first main step in proving the theorem is to replace the orientation condition by something more workable.

Proposition 0. Let $T: \boldsymbol{R}^{2} \rightarrow \boldsymbol{R}^{2}$ be a homeomorphism without fixed points. Then the following conditions are equivalent:

(a) $T$ preserves orientation.

(b) Let $C$ be any arcwise connected subset of $\boldsymbol{R}^{2}$. If TC $\cap C=\varnothing$ then $T^{n} C \cap C=\varnothing$ for all $n \neq 0$.

Proof. The implication (a) $\Rightarrow$ (b) is shown in [4, pp. 62-63] to be an elementary generalization of the following result of Brouwer:

LEMMA. Let $T$ be an orientation-preserving homeomorphism, without fixed points, of the plane $\boldsymbol{R}^{2}$ onto itself. Let $p \in \boldsymbol{R}^{2}$, and let $\alpha \subset \boldsymbol{R}^{2}$ be an arc with endpoints $p$ and Tp. Put $\alpha_{1}=\alpha-\{T p\}$. Then $\alpha_{1} \cap T \alpha_{1}=\varnothing$ implies $\alpha_{1} \cap T^{n} \alpha_{1}=\varnothing$ for all $n \neq 0$.

Brouwer's lemma and its proof appear in [1, pp. 38-40]. (The proof proceeds by a somewhat intricate computation of a certain winding number.)

Received by the editors July 3, 1969.

AMS Subject Classifications. Primary 5460, 5475, 5482, 5485.

Key Words and Phrases. Free mapping, flow line, edge point, edge point type, ray, side domain, regular domain. 
The implication (b) $\Rightarrow$ (a) is not vital to our purposes; it can, however, be deduced quite easily from the material in $\$ 2$.

In order to formalize the reduction of the problem which is achieved by Proposition 0 , we adopt the following definition.

Definition. A homeomorphism $T$ of the plane $\boldsymbol{R}^{2}$ onto itself is called a free mapping if

(1) $T$ has no fixed points.

(2) If $C$ is any arcwise connected subset of $R^{2}$ which satisfies $C \cap T C=\varnothing$, then $C \cap T^{n} C=\varnothing$ for all $n \neq 0$.

Throughout the paper we will be dealing with a single free mapping, which will always be denoted by $T$.

In the Appendix at the end of the paper we have listed, without proof, some of the basic facts about plane topology which we use.

1. Here, and in the succeeding section, we review some of the work of Brouwer in [1].

Proposition 1.1. Let $p \in \boldsymbol{R}^{2}$. Then $T^{n} p \rightarrow \infty$ as $n \rightarrow \pm \infty$.

Proof. Let $q \in \boldsymbol{R}^{2}$ be arbitrary; we show that $q$ is not an accumulation point for $\left\{T^{n} p\right\}$. Since $T q \neq q$, there is an open disc $D$ with $q \in D$ and $T D \cap D=\varnothing$. Since $T$ is free, we conclude that $T^{n} D \cap D=\varnothing$ for all $n \neq 0$.

Hence $D$ can contain no more than one point from the sequence $\left\{T^{n} p\right\}$. Therefore $q$ is not an accumulation point for $\left\{T^{n} p\right\}$. Q.E.D.

REMARK. The proposition implies that for no bounded set $E \subset R^{2}$ is it true that $T E \subset E$ or $T^{-1} E \subset E$.

Definition. A set $F \subset \boldsymbol{R}^{2}$ is called a flow line for $T$ if

(1) $T F=F$.

(2) $F$ is the image of a one-to-one continuous function sending $\boldsymbol{R}$ into $\boldsymbol{R}^{2}$.

Proposition 1.2. Flow lines exist.

Proof. Let $D \subset \boldsymbol{R}^{2}$ be an open disc which satisfies $D \cap T D=\varnothing$ and $\bar{D} \cap \mathrm{Cl}(T D)$ $\neq \varnothing$. Then there is a point $p$ on the boundary of $D$ whose image $T p$ is also on the boundary of $D$.

Let $m$ be any arc whose endpoints are $p$ and $T p$ and which satisfies $m-\{p, T p\} \subset D$. Then set $F=\bigcup \pm \infty T^{n} m$.

$F$ is, in an obvious way, a continuous image of $R$. One sees that $m_{1}=m-\{T p\}$ is disjoint from its first image under $T$; hence, by freeness, $F=\bigcup_{-\infty}^{+\infty} T^{n} m_{1}$ is a disjoint union. This shows that $F$ has no self-intersections.

Finally, $F$ obviously satisfies $T F=F$. Q.E.D.

REMARK. Let $G \subset \boldsymbol{R}^{2}$ be the graph of $y=(1 / x) \sin (1 / x)$ for $x>0$.

The set $G$ actually occurs as a flow line for a certain free mapping; it shows, for example, that a flow line need not be a closed subset of $\boldsymbol{R}^{2}$.

For the remainder of this section, $F$ will denote a single given flow line. 
Proposition 1.3. Let $C \subset R^{2}$ be arcwise connected and satisfy $C \cap T C=\varnothing$. Let $F \subset \boldsymbol{R}^{2}$ be a flow line, $p$ a point of $F$, and $m \subset F$ the closed arc from $p$ to $T p$ along $F$.

Suppose $C \cap m \neq \varnothing$, but that $C \cap T^{-1} m=\varnothing$ and $C \cap T m=\varnothing$.

Then $C \cap F=C \cap m$.

Proof. Put $m_{1}=m-\{T p\}$. Then $m_{1} \cap T m_{1}=\varnothing$, and $\bigcup_{-\infty}^{+\infty} T^{n} m_{1}=F$.

Now put $K=m_{1} \cup C$. Since $C \cap m_{1} \neq \varnothing, K$ is arcwise connected. Since the four sets $C \cap T C, m_{1} \cap T m_{1}, C \cap T^{-1} m_{1}$, and $C \cap T m_{1}$ are all empty, we have $K \cap T K=\varnothing$.

Therefore $K \cap T^{n} K=\varnothing$ for all $n \neq 0$. In particular, $C \cap T^{n} m_{1}=\varnothing$ for all $n \neq 0$. Since $F=\bigcup_{n} T^{n} m_{1}$, we have $C \cap F=C \cap m_{1}=C \cap m$. Q.E.D.

Proposition 1.4. Let $w$ be any subarc of the flow line $F$, and let $q$ be an interior point of $w$. Then there is an open set $D \subset R^{2}$ with $q \in D$ and $D \cap F=D \cap w$.

Proof. Choose $p \in F$ so that $q \in m$ where $m \subset F$ is the subarc determined by $p$ and $T p$. The sets $T^{-1} m$ and $T m$ are compact, and do not contain $p$. If $D$ is any disc centered at $p$ which avoids $T^{-1} m$ and $T m$ then it follows from Proposition 1.3 that $D \cap F=D \cap m$.

Let $D$ be chosen small enough so that $D \cap m=D \cap w$, as well as $D \cap T m$ $=D \cap T^{-1} m=\varnothing$. This $D$ satisfies the requirements of Proposition 1.4. Q.E.D.

Proposition 1.5. Let $F$ be a flow line, and let $C$ be a compact arcwise connected set which satisfies $C \cap \bar{F}=$ two points $=\{a, b\}$. Suppose in addition that $C \cap T C=\varnothing$. Then $a$ and $b$ are either both in $F$ or both in $\bar{F}-F$.

Proof. Suppose $a \in F$, and suppose if possible that $b \in \bar{F}-F$.

Then in particular $C \cap F=\{a\}$.

Choose $p \in F$ so that $a \in m$, where $m \subset F$ is the subarc determined by $p$ and $T p$. Then $C \cap m \neq \varnothing$ while $C \cap T^{-1} m=\varnothing$ and $C \cap T m=\varnothing$.

Because the relevant sets are compact, there exists a connected open set $\mathcal{O}$, containing $C$, such that $\mathcal{O} \cap T \mathcal{O}, \mathcal{O} \cap T^{-1} m$, and $\mathcal{O} \cap T m$ are all empty.

By Proposition 1.3, $\mathcal{O} \cap F=\mathcal{O} \cap m$. Hence $\mathcal{O} \cap \bar{F}=\mathcal{O} \cap \bar{m}=\mathcal{O} \cap m$. But this is impossible since $\mathcal{O}$ contains $b$, where $b \in \bar{F}$ but $b \notin F$. Q.E.D.

Notation. $D_{r}(p)$ is the open disc with radius $r>0$ and center $p \in \boldsymbol{R}^{2}$.

Definition. A point $p \in \boldsymbol{R}^{2}-\bar{F}$ is called an edge point of $F$ if, for some $r>0$, we have $D_{r}(p) \cap F \neq \varnothing$ and $D_{r}(p) \cap T\left(D_{r}(p)\right)=\varnothing$.

Definition. Let $p$ be an edge point of $F$, and let $D_{r}(p)$ satisfy $D_{r}(p) \cap F \neq \varnothing$ and $D_{r}(p) \cap T\left(D_{r}(p)\right)=\varnothing$. Then there exists a compact arcwise connected set $C$, $p \in C \subset D_{r}(p)$, with $C \cap \bar{F}=\{$ one point $\}=a$. Then $p$ is of type $F$ or type $\bar{F}-F$ according as $a \in F$ or $a \in \bar{F}-F$.

Proposition 1.6. Edge point type is well-defined.

Proof. Suppose that $C_{1}$ and $C_{2}$ are compact arcwise connected sets with $p \in C_{1} \cap C_{2}$ and $C_{i} \cap \bar{F}=\left\{a_{i}\right\}$ for $i=1,2$. If the $C_{i}$ arise from the definition of edge 
point type, then each $C_{i}$ is contained in a disc $D_{r_{i}}(p)$ which satisfies $D_{r_{i}}(p) \cap$ $T\left(D_{r_{i}}(p)\right)=\varnothing$. Put $K=C_{1} \cup C_{2}$; we have $K \cap T K=\varnothing$ because $K$ is contained in the larger of the two discs. $K$ is connected since $C_{1} \cap C_{2} \neq \varnothing$; we also have $K \cap \bar{F}=\left\{a_{1}, a_{2}\right\}$.

By Proposition 1.5, $a_{1}$ and $a_{2}$ are both in $F$ or both in $\bar{F}-F$. Q.E.D.

REMARK. It is easy to see that the set of all edge points of $F$ is open, and that the edge point type is constant on the connected components of this open set.

Proposition 1.7. Let $\alpha$ be an arc with endpoints $p$ and $q$. Suppose $\alpha \cap \bar{F}=\{p, q\}$. Then $p$ and $q$ are either both in $F$ or both in $\bar{F}-F$.

Proof. We begin by assuming that $\alpha$ is a polygonal arc.

Suppose $p \in F$ and $q \in \bar{F}-F$. Choose $r>0$ so that $D_{4 r}(q) \cap \alpha$ is a straight line segment, and $D_{4 r}(q) \cap T\left(D_{4 r}(q)\right)=\varnothing$.

Choose $a \in F$ so that the arc $F_{1} \subset F$ determined by $p$ and $a$ satisfies $F_{1} \cap \mathrm{Cl}\left(D_{r}(q)\right)$ $=\{a\}$. Let $\sigma$ be the straight line segment with endpoints $a$ and $q$; then $\Gamma=\alpha \cup \sigma \cup F_{1}$ is a Jordan curve.

Put $K=\sigma \cup \mathrm{Cl}(F \cap$ int $\Gamma) ; K$ is compact and connected. Moreover, we claim that $K \cap \Gamma=\sigma$. Indeed, $K \cap \alpha=\{q\}$ by the assumption that $\alpha \cap \bar{F}=\{p, q\}$, and $K \cap F_{1}=\{a\}$ by an application of Proposition 1.4.

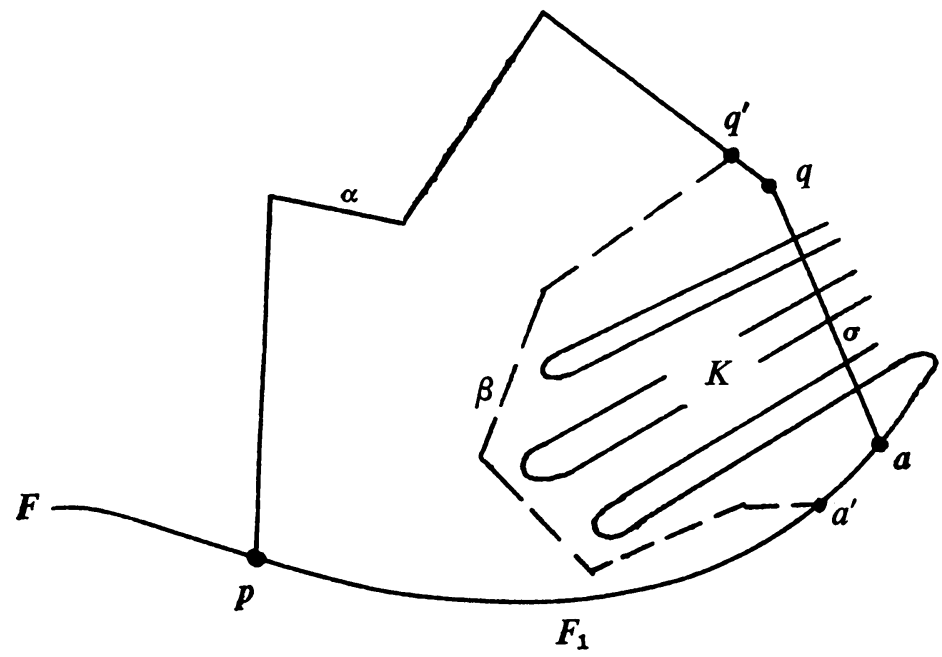

Now choose $\varepsilon$ so that $0<\varepsilon<r$, and $D_{\varepsilon}(x) \cap T\left(D_{\varepsilon}(x)\right)=\varnothing$ for all $x \in \mathrm{Cl}$ (int $\Gamma$ ). By App. 5, there is an arc $\beta$, with endpoints $q^{\prime}$ and $a^{\prime}$, so that

(1) $\beta-\left\{a^{\prime}, q^{\prime}\right\} \subset$ (int $\left.\Gamma\right)-K$.

(2) $a^{\prime} \in F_{1}-\sigma$ and $q^{\prime} \in \alpha-\sigma$.

(3) $D_{\varepsilon}(x)$ meets $K$ for all $x \in \beta$.

It follows that $\beta \cap \bar{F}=\varnothing$. Furthermore, each point $x$ of $\beta$ is an edge point of $F$ : 
if $D_{\varepsilon}(x)$ meets $F$ this claim is obvious; if $D_{\varepsilon}(x)$ meets $\sigma$ then $D_{2 r}(x)$ will meet $q \in \bar{F}$ and still satisfy $D_{2 r}(x) \cap T\left(D_{2 r}(x)\right)=\varnothing$.

Hence all the points of $\beta$ are edge points. But $q^{\prime} \in \beta$ is of type $\bar{F}-F$, while points of $\beta$ near $a^{\prime}$ are obviously of type $F$. By the Remark following Proposition 1.6, we have reached a contradiction. This completes the proof for the case where $\alpha$ is polygonal.

Suppose now that $\alpha$ is a general arc. Because of the local constancy of edge point type, there would exist a polygonal arc $\alpha^{\prime}$, with endpoints $p^{\prime}$ and $q^{\prime}$, such that $\alpha^{\prime} \cap \bar{F}=\left\{p^{\prime}, q^{\prime}\right\}$ with $p^{\prime} \in F$ and $q^{\prime} \in \bar{F}-F$. This is the case just treated, hence the proof is complete. Q.E.D.

For the remainder of this section we consider a single flow line $F$, and a chosen subarc $m \subset F$ which satisfies $m \cap T m \neq \varnothing$.

Let $m_{0}=m-\{u, v\}$ where $u$ and $v$ are the endpoints of $m$.

Definition. The rays of $F$ are the two arcwise connected components of $F-m_{0}$.

Proposition 1.8. Let $f \subset F$ be a ray. Then $F \cap \bar{f}=f$.

Proof. Let $q \in F-f$. Then $q$ is in the interior of a subarc $\beta$ of $F$ which satisfies $\beta \cap f=\varnothing$. By Proposition $1.4, q$ is contained in a disc $D$ which satisfies $D \cap F$ $=D \cap \beta$; hence in particular $D \cap f=\varnothing$.

Thus $D \cap \bar{f}=\varnothing$, and we have shown that $q \notin \bar{f}$. Q.E.D.

From now on we denote the two rays of $F$ by $i$ and $f$; for definiteness let the notation be chosen so that $i \subset T i$ and $f \supset T f$.

Proposition 1.9. Let $C$ be an arcwise connected set which satisfies $C \cap \bar{F}=$ two points $=\{a, b\}$. Suppose in addition that $C \cap T C=\varnothing$. Then it is impossible that $a \in i$ and $b \in f$.

Proof. Suppose $a \in i$ and $b \in f$. Then it is possible to choose $p \in F$ so that $\left\{p, a, T p, T^{2} p, b\right\}$ is the order in which these five points occur along $F$.

Let $\alpha \subset F$ be the closed subarc determined by $p$ and $T p$. Then $C \cap \alpha \neq \varnothing$ while $C \cap T^{-1} \alpha$ and $C \cap T \alpha$ are both empty. By Proposition 1.3, $C \cap F=C \cap \alpha=\{a\}$. But this contradicts $b \in C \cap F$. Q.E.D.

RemarK. Proposition 1.9 affords the following refinement of edge point type. Let $p$ be an edge point of $F$, of type $F$. From the definition, there is an open disc $D$ centered at $p$ so that $D \cap F \neq \varnothing$ and $D \cap T D=\varnothing$.

But suppose $D$ can be chosen so that it also satisfies $D \cap m=\varnothing$. Then $p$ is of type $i$ or type $f$, according as the set $C$ in the definition of edge point type meets $i$ or $f$.

This "refined" edge point type is well defined, by a straightforward application of Proposition 1.9. Moreover, it is locally constant wherever it is defined, i.e., on those points $p$ of $\boldsymbol{R}^{2}-\bar{F}$ which admit open discs $D_{r}(p)$ satisfying $D_{r}(p) \cap F \neq \varnothing$, $D_{r}(p) \cap T\left(D_{r}(p)\right)=\varnothing$, and $D_{r}(p) \cap m=\varnothing$.

Proposition 1.10. $i \cap \bar{f}=\varnothing$. 
Proof. Suppose $p \in i \cap \bar{f}$. Then in particular $p \notin m$. Choose $r>0$ so that $D_{4 r}(p)$ avoids $m$ and satisfies $D_{4 r}(p) \cap T\left(D_{4 r}(p)\right)=\varnothing$.

Choose $x \in i$ and $y \in f$ so that the subarc $F_{1} \subset F$ they determine satisfies $F_{1} \cap \mathrm{Cl}\left(D_{r}(p)\right)=\{x, y\}$. Let $\sigma$ be the straight line segment determined by $x$ and $y$. Then $\Gamma=\sigma \cup F_{1}$ is a Jordan curve.

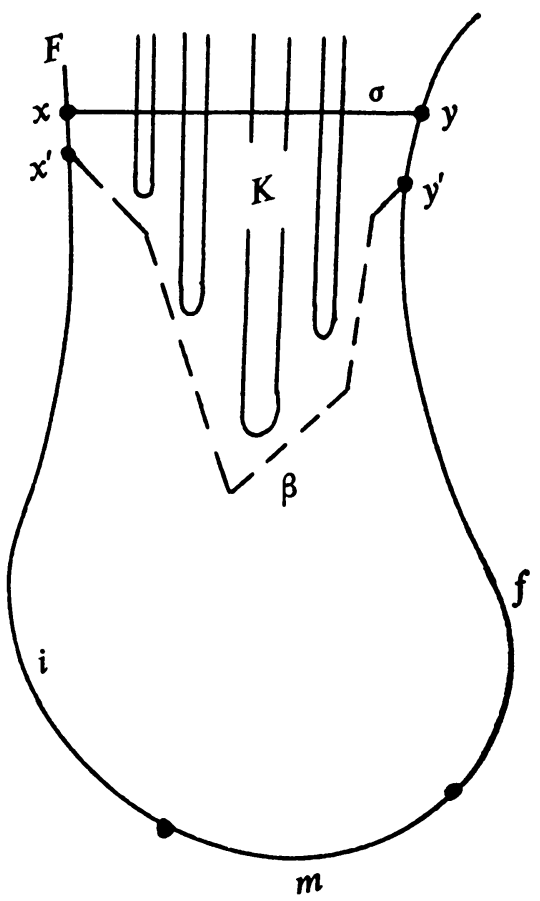

Put $K=\sigma \cup \mathrm{Cl}(F \cap$ int $\Gamma)$. Then $K$ is compact, connected, and satisfies $K \cap \Gamma=\sigma$.

Let $\eta$ denote the distance from $K$ to $m$. Choose $\varepsilon$ so that $0<\varepsilon<r, \varepsilon<\frac{1}{2} \eta$, and $D_{\varepsilon}(q) \cap T\left(D_{\varepsilon}(\bar{q})\right)=\varnothing$ for all $q \in \mathrm{Cl}$ (int $\Gamma$ ).

By App. 5, there is an $\operatorname{arc} \beta$ with endpoints $x^{\prime}$ and $y^{\prime}$, such that

(1) $\beta-\left\{x^{\prime}, y^{\prime}\right\} \subset$ (int $\left.\Gamma\right)-K$.

(2) $x^{\prime} \in i-\sigma$ and $y^{\prime} \in f-\sigma$.

(3) $D_{\varepsilon}(z) \cap K \neq \varnothing$ for all $z \in \beta$.

Now each point $z \in \beta$ is the center of some disc $D$ which satisfies $D \cap F \neq \varnothing$, $D \cap m=\varnothing$, and $D \cap T D=\varnothing$. Indeed, if $D_{\varepsilon}(z)$ meets $F$, then $D_{\varepsilon}(z)$ itself will do. Otherwise, by (3), $D_{\delta}(z)$ meets $\sigma$; in this case $D_{2 r}(z)$ will do.

Consequently the refined edge point type is defined for all points of $\beta$. By inspection, $\beta$ contains points of type $i$ and of type $f$. Therefore we have reached a contradiction to the Remark following Proposition 1.9. Q.E.D.

Proposition 1.11. Let $\alpha$ be an arc with endpoints $p$ and $q$. Suppose $\alpha \cap \bar{F}=\{p, q\}$. If $p, q \in \bar{F}-F$, then $p$ and $q$ are either both in $i$ or both in $\bar{f}$. 
Proof. Suppose $p \in \bar{i}$ and $q \in \bar{f}$. Because $i \cap \bar{f}=\varnothing$, one can choose $r>0$ such that (1) $D_{r}(p) \cap \alpha$ and $D_{r}(q) \cap \alpha$ are straight line segments.

(2) $D_{r}(p)$ avoids $m \cup \bar{f}$, and $D_{r}(q)$ avoids $m \cup i$.

Let $x \in i$ and $y \in f$ be chosen so that the subarc $F_{1} \subset F$ they determine satisfies $F_{1} \cap \mathrm{Cl}\left(D_{r}(p)\right)=\{x\}$ and $F_{1} \cap \mathrm{Cl}\left(D_{r}(q)\right)=\{y\}$. Let $\sigma_{p}$ and $\sigma_{q}$ be the straight line segments determined by $p$ and $x, q$ and $y$, resp. Finally put $\Gamma=\alpha \cup \sigma_{p} \cup \sigma_{q} \cup F_{1}$; it is a Jordan curve.

Put $K_{i}=\sigma_{p} \cup \mathrm{Cl}(i \cap$ int $\Gamma)$ and $K_{f}=\sigma_{q} \cup \mathrm{Cl}(f \cap$ int $\Gamma)$. The choice of $r$, together with the fact that $i \cap \bar{f}=\varnothing$, implies that $K_{i} \cap K_{f}=\varnothing$. Moreover $K_{i} \cap \Gamma=\sigma_{i}$ and $K_{f} \cap \Gamma=\sigma_{f}$; this follows from Proposition 1.4, the argument being the same as in the proof of Proposition 1.7.

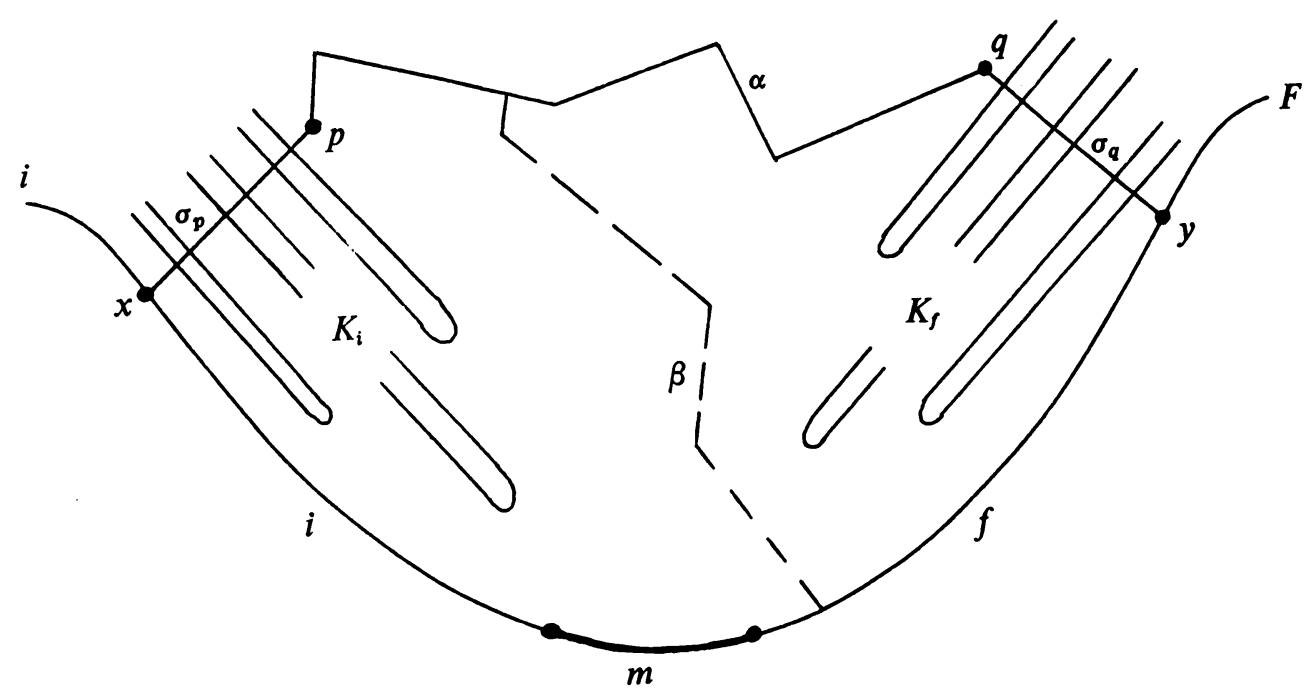

By App. 4 there exists a polygonal arc $\beta$ such that $\beta \cap\left(K_{i} \cup K_{f}\right)=\varnothing, \beta \cap$ ext $\Gamma$ $=\varnothing$, and $\beta$ meets both components of $\Gamma-\left(K_{i} \cup K_{f}\right)$. But now $\beta$ followed by $\alpha$ would give an arc $\gamma$ which violated Proposition 1.7; except for its endpoints, $\gamma$ would avoid $\bar{F}$, and one endpoint would be in $F$ while the other would be in $\bar{F}-F$. This completes the proof for polygonal $\alpha$.

The extension to general arcs $\alpha$ is deduced from the polygonal case in the same way as in the proof of Proposition 1.7; one uses here the fact that $i \cap \bar{f}=\varnothing$. Q.E.D.

The following is a summary of the results of $\S 1$.

Summary. Let $F$ be a flow line, $m \subset F$ a closed subarc satisfying $m \cap T m \neq \varnothing$, and let $f, i \subset F$ be the associated rays.

Let $\alpha \subset \boldsymbol{R}^{2}$ be an arc with endpoints $p$ and $q$; suppose that $\alpha \cap \bar{F}=\{p, q\}$.

Then exactly one of the following three statements is true:

(1) $p$ and $q$ are both in $F$.

(2) $p$ and $q$ are both in $i-F$. 
(3) $p$ and $q$ are both in $\bar{f}-F$.

In particular, $i \cap \bar{f}=\varnothing$.

2. Let $F$ be a flow line. To minimize technicalities, we assume that $F$ arises in the following way.

Let $D$ be an open disc which satisfies $D \cap T D=\varnothing$ and $\bar{D} \cap \mathrm{Cl}(T D) \neq \varnothing$. Then the boundary of $D$ contains a pair of points of the form $\{p, T p\}$. Let $m$ be a polygonal arc, with endpoints $p$ and $T p$, which satisfies $m-\{p, T p\} \subset D$. Then we shall consider the flow line $F=\bigcup \bigcup_{-\infty}^{+\infty} T^{n} m$.

Definition. A component $\Omega$ of $\boldsymbol{R}^{2}-\bar{F}$ is called a side domain of $F$ if there exists a line segment $\sigma$ with endpoint $q$ such that $q \in F$ and $\sigma-\{q\} \subset \Omega$.

Proposition 2.1. There exist at least two side domains.

Proof. Let $D_{1}$ and $D_{2}$ be the two components of $D-m$. Since $D_{n} \cap \bar{F}=m, D_{1}$ and $D_{2}$ determine side domains $\Omega_{1}$ and $\Omega_{2}$. If $\Omega_{1}$ and $\Omega_{2}$ were the same, a polygonal Jordan curve $\Gamma$ would exist which intersected $F$ transversally in a single point of $m$. Then one of the two components of $F-\Gamma$ would be contained in int $\Gamma$. This, however, is impossible because int $\Gamma$ is bounded while both components of $F-\Gamma$ are unbounded. Q.E.D.

Proposition 2.2. The two side domains which are determined by the components of $D-m$ are invariant under $T$.

Proof. Let $D_{1}$ be one of the components of $D-m$, and let $\Omega \supset D_{1}$ be the side domain it determines.

Let $i$ and $f$ be the components of $F-m$. There exists an arc $\alpha$, with endpoints $a$ and $b$, such that $\alpha \cap D_{1} \neq \varnothing$ and $\alpha \cap \bar{F}=\{a, b\}$, with $a \in i$ and $b \in f$. Now $\alpha-\{a, b\} \subset \Omega$. By Proposition 1.8 we have $\alpha \cap T \alpha \neq \varnothing$. Thus, in particular, $\Omega \cap T \Omega \neq \varnothing$.

Now $\Omega$ is a component of $R^{2}-\bar{F}$; hence either $\Omega \cap T \Omega=\varnothing$ or $\Omega=T \Omega$. The first possibility is the one we have just ruled out. Q.E.D.

Proposition 2.3. F has precisely two side domains, and each is invariant under $T$.

Proof. Let $\Omega$ be an arbitrary side domain of $F$. Then there is an $\operatorname{arc} \gamma$, with endpoint $q \in F$, such that $\gamma-\{q\} \subset \Omega$. We can assume without loss of generality that $q$ is not an iterate of $p$; since $\lim _{n \rightarrow \infty} T^{n} p=\infty$, the original $\gamma$ could be diverted slightly so that it meets $F$ in $F-\cup T^{n} p$.

Thus, some point of $m-\{p, T p\}$ is a boundary point of $T^{n} \Omega$ for some integer $n$. Hence $T^{n} \Omega$ meets $D_{1}$ or $D_{2}$ and hence $T^{n} \Omega=\Omega_{1}$ or $\Omega_{2}$. By Proposition 2.2 we have $\Omega=\Omega_{1}$ or $\Omega_{2}$. Q.E.D.

Proposition 2.4. Let $\Omega$ be a side domain of $F$. Let $\sigma$ be a curve with endpoint $p$, such that $\sigma-\{p\} \subset \Omega$ and $p \in \bar{F}$. Then actually $p \in F$. 
Proof. By the definition of side domain, one can extend $\sigma$ to an arc $\alpha$, with endpoints $p$ and $q$, where $\alpha \cap \bar{F}=\{p, q\}$ and $q \in F$. The conclusion now follows by the result of $\S 1$. Q.E.D.

THEOREM 2.5. Let $\Gamma$ be a polygonal Jordan curve with $m \subset$ int $\Gamma$. Let $\Omega$ be a side domain of $F$. Then $\Gamma$ contains a subarc $\gamma$, with endpoints $a$ and $b$, such that $\gamma \cap \bar{F}$ $=\{a, b\}, \gamma-\{a, b\} \subset \Omega, a \in i$, and $b \in f$.

Proof. Since $m \subset$ int $\Gamma$, we have $\Gamma \cap \bar{F}=(\Gamma \cap \bar{\imath}) \cup(\Gamma \cap \bar{f})$. Say a closed subarc $I$ of $\Gamma$ is crossing if $I$ meets both $i$ and $\bar{f}$, but no proper subarc of $I$ meets $i$ and $\bar{f}$. Because $i \cap \bar{f}=\varnothing$, there is a nonzero distance from $\Gamma \cap i$ to $\Gamma \cap \bar{f}$; hence there are only finitely many crossing subarcs. Let them be denoted by $I_{1}, I_{2}, \ldots, I_{k}$.

Let $I_{k}$ be a typical crossing arc, with endpoints $a$ and $b$. We have $I_{k} \cap \bar{F}=\{a, b\}$ with $a \in i$ and $b \in \bar{f}$. But by Proposition 1.11, we actually have $a \in i$ and $b \in f$. Thus, the theorem will be proved once we show that among the $\left\{I_{k}\right\}$ there is one that meets $\Omega$.

Put $I=\bigcup_{k=1}^{N} I_{k}$. The components of $\Gamma-I$ are open subarcs; let $J$ be the closure of a typical such subarc. Then $J$ cannot meet both $i$ and $\bar{f}$, for otherwise a proper subarc of $J$ would be among the $\left\{I_{k}\right\}$. On the other hand, $J$ does meet $i \cup \bar{f}$, because of the definition of crossing arc.

Thus the components of $\Gamma-I$ are classified according to whether their closures meet $i$ or $\bar{f}$. Let $J^{i} \subset \Gamma$ be the union of those $J$ 's which meet $i$; in like manner define $J^{f} \subset \Gamma$.

One has the relations $I \cup J^{i} \cup J^{f}=\Gamma$ and $\left(i \cup J^{i}\right) \cap\left(\bar{f} \cup J^{f}\right)=\varnothing$.

Now choose closed subarcs $F^{i} \subset i$ and $F^{f} \subset f$; let them be large enough so that they include all endpoints of all crossing arcs. Put $C^{i}=F^{i} \cup J^{i}, C^{f}=F^{f} \cup J^{f}$, and $K=F^{i} \cup F^{f} \cup I$. Then $C^{i}, C^{f}$, and $K$ are compact connected sets.

Now $C^{i} \cap C^{f}=\varnothing$, while $C^{i} \cap K$ and $C^{f} \cap K$ are both connected. Indeed, $C^{i} \cap K=F^{i} \cap K=F^{i}$, a subarc of the flow line $F$.

Let $q$ be a point of $m$; we wish to show that $q \in$ int $(K)$ where in general the notation $q \in$ int $(C)$ means that $q$ is in a bounded component of the complement of the closed set $C$.

Now, $q \in \operatorname{ext} C^{f}$, since $q$ is on the unbounded set $i \cup m$ which avoids $C^{f}$. Likewise $q \in$ ext $C^{i}$.

Now suppose, if possible, that $q \in \operatorname{ext} K$. Since $K \cap C^{f}$ is connected, we could conclude by App. 2 that $q \in \operatorname{ext} K \cup C^{f}$. But $\left(K \cup C^{f}\right) \cap C^{i}$ is also connected; hence a second application of App. 2 would lead to the conclusion that

$$
q \in \operatorname{ext}\left(K \cup C^{f} \cup C^{i}\right) \text {. }
$$

This is impossible because $K \cup C^{f} \cup C^{i}$ contains $\Gamma$ and we already know that $q \in$ int $\Gamma$.

Thus $q \in$ int $K$. It now follows that $I \cap \Omega \neq \varnothing$; for otherwise the unbounded connected set $\Omega$ would avoid $K$, and consequently points arbitrarily close to $q$ would be in ext $K$. 


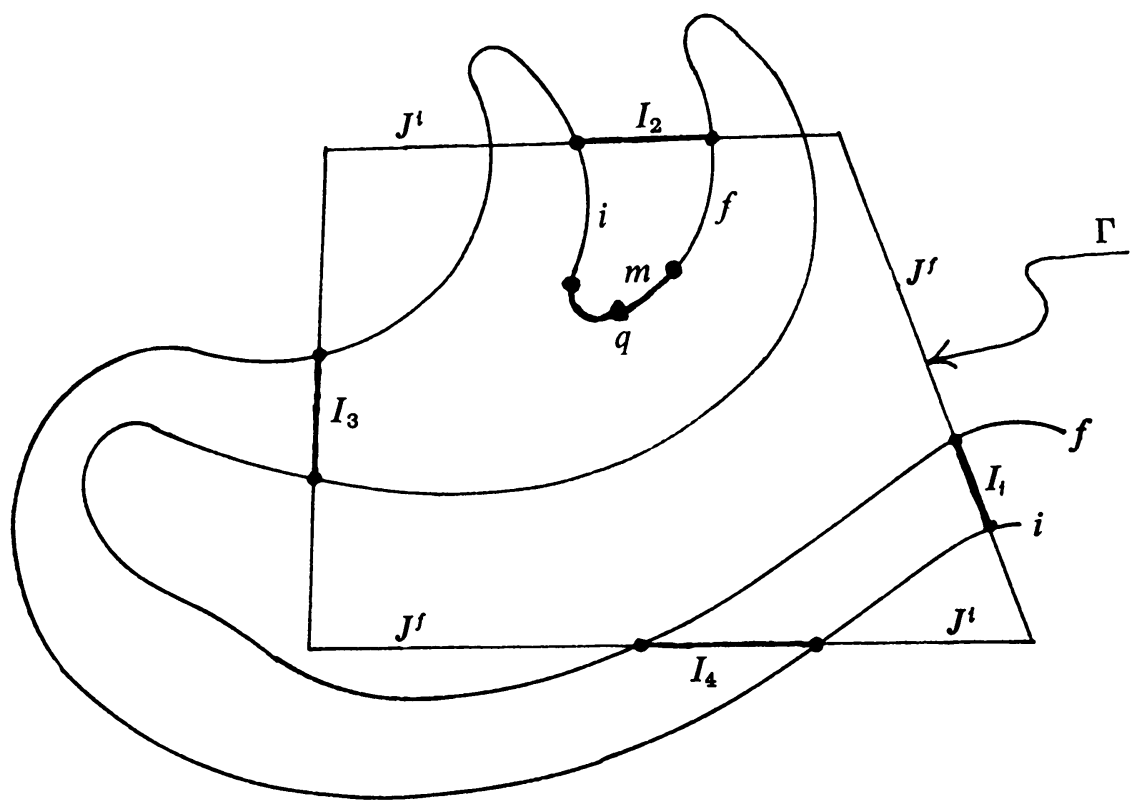

Hence $I_{k} \cap \Omega \neq \varnothing$ for some crossing arc $I_{k}$. This completes the proof of the theorem. Q.E.D.

3. Throughout the present section $F_{1}$ and $F_{2}$ will be two flow lines which satisfy $F_{1} \cap F_{2}=\varnothing$.

DEFINITION. An arc $\alpha$ with endpoints $a_{1}$ and $a_{2}$ is called a crosscut if $\alpha \cap F_{n}=\left\{a_{n}\right\}$ for $n=1,2$.

Proposition 3.1. Suppose $F_{1}$ and $F_{2}$ have a crosscut $\alpha$. Then $\bar{F}_{1} \cap F_{2}=F_{1} \cap \bar{F}_{2}=\varnothing$, and $\alpha \cap \mathrm{Cl}\left(F_{n}\right)=\left\{a_{n}\right\}$, for $n=1,2$.

Proof. By hypothesis $\left(\alpha \cup F_{1}\right) \cap F_{2}=\left\{a_{2}\right\}$.

Since $\alpha \cup F_{1}$ is arcwise connected it meets $\mathrm{Cl}\left(F_{2}\right)$ only in the single point $a_{2}$, for otherwise $\alpha \cup F_{1}$ would contain an arc which violated Proposition 1.7. Q.E.D.

Definition. Let $\alpha$ and $\beta$ be crosscuts. We define $K(\alpha, \beta)$ to be the set $\alpha \cup \beta$ $\cup S_{1} \cup S_{2}$, where $S_{n} \subset F_{n}$ is the subarc determined by the endpoints of $\alpha$ and $\beta$.

REMARK. $K(\alpha, \beta)$ is not in general a Jordan curve, since $\alpha \cap \beta \neq \varnothing$ is still possible. But the notation int $K(\alpha, \beta)$ will still be employed to designate any bounded component of $\boldsymbol{R}^{2}-K(\alpha, \beta)$.

The following is the main result of this section.

THeOREM 3. Let $\alpha$ be a polygonal crosscut which satisfies $\alpha \cap T \alpha=\varnothing$. Let $p$ be a point of $\alpha$.

Let $\beta$ be any other crosscut. Then for certain integers $r$ and $s$ we have

$$
p \in \operatorname{int} K\left(T^{r} \beta, T^{s} \beta\right) .
$$


The proof of Theorem 3 begins with the following facts. (We use $F_{n}(a)$ to designate the rays of a flow line $F$ which are determined by a point $a \in F$.)

Proposition 3.2. Let $F_{1}\left(a_{1}\right) \subset F_{1}$ and $F_{2}\left(a_{2}\right) \subset F_{2}$ be any choice of rays. Then $T^{-n} p$ and $T^{n} p$ are separated by $\mathrm{Cl}\left(F_{1}\left(a_{1}\right)\right) \cup \alpha \cup \mathrm{Cl}\left(F_{2}\left(a_{2}\right)\right)$ for every $n>0$.

Proof. Since $T \alpha \cap \alpha=\varnothing$, we have that $K\left(T^{-n} \alpha, \alpha\right)$ and $K\left(\alpha, T^{n} \alpha\right)$ are both Jordan curves; moreover their intersection is just $\alpha$.

By App. 6, their interior domains are either disjoint or nested. But $T^{n}$ sends int $K\left(T^{-n} \alpha, \alpha\right)$ onto int $K\left(\alpha, T^{n} \alpha\right)$; hence the interiors cannot be nested or else we would have a bounded set taken into itself by $T^{n}$.

Therefore int $K\left(T^{-n} \alpha, \alpha\right) \cap$ int $K\left(\alpha, T^{n} \alpha\right)=\varnothing$. Consequently, if $\sigma$ is a short line segment which meets $\alpha$ transversally in a single point, then $\sigma$ meets both int $K\left(T^{-n} \alpha, \alpha\right)$ and int $K\left(\alpha, T^{n} \alpha\right)$.

It follows that int $K\left(T^{-n} \alpha, \alpha\right)$ and int $K\left(\alpha, T^{n} \alpha\right)$ are separated by $\mathrm{Cl}\left(F_{1}\left(a_{1}\right)\right) \cup \alpha$ $\cup \mathrm{Cl}\left(F_{1}\left(a_{2}\right)\right)$; for otherwise there would exist a polygonal Jordan curve $\Gamma$ whose intersection with $\mathrm{Cl}\left(F_{1}\left(a_{1}\right)\right) \cup \alpha \cup \mathrm{Cl}\left(F_{2}\left(a_{2}\right)\right)$ would consist precisely of the transversal intersection $\sigma \cap \alpha$. But this is absurd because one of the two unbounded sets $F_{n}\left(a_{n}\right)$ would then lie in int $\Gamma$.

Now the points $T^{-n} p$ and $T^{n} p$ are, respectively, in the same components of $\boldsymbol{R}^{2}-\left(\mathrm{Cl}\left(F_{1}\left(a_{1}\right)\right) \cup \alpha \cup \mathrm{Cl}\left(F_{2}\left(a_{2}\right)\right)\right)$ as int $K\left(T^{-n} \alpha, \alpha\right)$ and int $K\left(\alpha, T^{n} \alpha\right)$. Hence they too are separated by $\mathrm{Cl}\left(F_{1}\left(a_{1}\right)\right) \cup \alpha \cup \mathrm{Cl}\left(F_{2}\left(a_{2}\right)\right)$. Q.E.D.

Proposition 3.3. Let $\alpha$ and $\beta$ be any crosscuts.

Put $C=\alpha \cup \mathrm{Cl}\left(F_{1}\left(a_{1}\right)\right) \cup \mathrm{Cl}\left(F_{2}\left(a_{2}\right)\right)$, where the $F_{n}\left(a_{n}\right) \subset F_{n}$ are any choice of rays emanating from the endpoints of $\alpha$.

Then $C \cap K(\alpha, \beta)$ is connected.

Proof. First $K(\alpha, \beta)=\alpha \cup \beta \cup S_{1} \cup S_{2}$, where $S_{n} \subset F_{n}$ is the subarc determined by the endpoints $a_{n}$ and $b_{n}$ of $\alpha, \beta$, respectively.

By Proposition 3.1, $\mathrm{Cl}\left(F_{1}\right)$ meets $\alpha \cup \beta \cup F_{2}$ only in the points $a_{1}$ and $b_{1} \in F_{1}$. Therefore $\mathrm{Cl}\left(F_{1}\right) \cap K(\alpha, \beta)=S_{1}$, and hence $\mathrm{Cl}\left(F_{1}\left(a_{1}\right)\right) \cap K(\alpha, \beta)=S_{1} \cap \mathrm{Cl}\left(F_{1}\left(a_{1}\right)\right)$. But we know from Proposition 1.8 that $\mathrm{Cl}\left(F_{1}\left(a_{1}\right)\right) \cap F_{1}=F_{1}\left(a_{1}\right)$; therefore

$$
\mathrm{Cl}\left(F_{1}\left(a_{1}\right)\right) \cap K(\alpha, \beta)=S_{1} \cap F_{1}\left(a_{1}\right) .
$$

Thus $C \cap K(\alpha, \beta)=\alpha \cup\left(S_{1} \cap F_{1}\left(a_{1}\right)\right) \cup\left(S_{2} \cap F_{2}\left(a_{2}\right)\right)$. Being, therefore, the union of $\alpha$ with two arcs that meet $\alpha, C \cap K(\alpha, \beta)$ is arcwise connected. Q.E.D.

Proposition 3.4. There exists $N_{0}>0$ such that, for all $n>N_{0}$, the points $T^{-n} p$ and $T^{n} p$ are separated by the set $C=\beta \cup \mathrm{Cl}\left(F_{1}\left(b_{1}\right)\right) \cup \mathrm{Cl}\left(F_{2}\left(b_{2}\right)\right)$, where the $F_{i}\left(b_{i}\right) \subset F_{i}$ are any choice of rays.

Proof. Set $K=K(\alpha, \beta)$. Choose $N_{0}>0$ so that $n \geqq N_{0}$ implies that $T^{-n} p$ and $T^{n} p$ are not separated by $K$. This is possible because $K$ is bounded and $T^{n} p \rightarrow \infty$ as $n \rightarrow \pm \infty$. 
We prove the lemma for all $n \geqq N_{0}$. Suppose, if possible, that $T^{n} p$ and $T^{-n} p$ were not separated by $C$. Now $K$ is compact, $C$ is closed, and $C \cap K$ is connected by Proposition 3.3. Therefore, by App. 2, $T^{-n} p$ and $T^{n} p$ would not be separated by $C \cup K$.

But $C \cup K$ contains a set of the form $\alpha \cup \mathrm{Cl}\left(F_{1}\left(a_{1}\right)\right) \cup \mathrm{Cl}\left(F_{2}\left(a_{2}\right)\right)$. By Proposition $3.2, T^{-n} p$ and $T^{n} p$ are separated by any such set; therefore they must also be separated by the larger set $C \cup K$.

This completes the proof. Q.E.D.

Proposition 3.5. There exist integers $u$ and $v$, and a choice of rays $F_{1}\left(b_{1}\right) \subset F_{1}$ and $F_{2}\left(b_{2}\right) \subset F_{2}$, such that the points $T^{u} p$ and $T^{v} p$ are not separated by

$$
\beta \cup \mathrm{Cl}\left(F_{1}\left(b_{1}\right)\right) \cup \mathrm{Cl}\left(F_{2}\left(b_{2}\right)\right) .
$$

Furthermore, the choice of integers and rays can be made so that $u-v=2 k$, where $k$ is an integer larger than any preassigned $N>0$.

Proof. Let $D$ be an open disc which contains $\beta$. Let $k$ be any integer which is large enough so that $n>k$ implies $T^{n} p \notin \bar{D}$.

Our integers $u$ and $v$ will be chosen from the set $\Delta=\{2 k, 4 k, 6 k, 8 k, 10 k\}$. (The condition that $u-v$ be a large even integer will obviously be satisfied.)

Consider a given $n \in \Delta$. Since $T^{n} p \notin \bar{D}$, there exists a polygonal arc $\gamma$, with endpoints $T^{n} p$ and $q$, such that $\gamma \cap \beta=\varnothing$ and $\gamma \cap\left(\bar{F}_{1} \cup \bar{F}_{2}\right)=\{q\}$.

By Proposition 3.1, $p \in \Omega_{1} \cap \Omega_{2}$, where $\Omega_{k}$ is that side domain of $F_{k}$ which contains $\alpha$. Hence, by invariance of side domains, the arc $\gamma$ lies, except for its endpoint $q$, in $\Omega_{1} \cap \Omega_{2}$. By Proposition 2.3, $q$ actually lies in $F_{1} \cup F_{2}$, and thus lies on one of the four rays determined by the points $b_{k} \in F_{k}$ for $k=1,2$.

Now $\Delta$ has five elements while there are only four possible rays. Hence let $u$ and $v \in \Delta$ be chosen so that $T^{u} p$ and $T^{v} p$ have connecting arcs $\gamma_{u}$ and $\gamma_{v}$ which run to the same ray.

Now choose rays $F_{1}\left(b_{1}\right) \subset F_{1}$ and $F_{2}\left(b_{2}\right) \subset F_{2}$ so that $F_{1}\left(b_{1}\right) \cup F_{2}\left(b_{2}\right)$ does not meet $\gamma_{u} \cup \gamma_{v}$. This is possible because only one of the four possible rays meets $\gamma_{u} \cup \gamma_{v}$.

Thus $T^{u} p$ is connected to $T^{v} p$ by the arc $\gamma_{u} \cup S \cup \gamma_{v}$, where $S$ is a subarc of one of the flow lines; by construction $F_{1}\left(b_{1}\right) \cup \beta \cup F_{2}\left(b_{2}\right)$ is disjoint from

$$
\gamma_{u} \cup S \cup \gamma_{v}
$$

To complete the proof we must check that $\mathrm{Cl}\left(F_{1}\left(b_{1}\right)\right) \cup \mathrm{Cl}\left(F_{2}\left(b_{2}\right)\right) \cup \beta$ avoids $\gamma_{u} \cup \gamma_{v} \cup S$; but this is easily deduced from Propositions 3.1 and 1.8. Q.E.D.

Proof of Theorem 3. Let $N>0$ be chosen so that $n>N$ implies that $T^{n} p$ and $T^{-n} p$ are separated by $\beta \cup \mathrm{Cl}\left(F_{1}\left(b_{1}\right)\right) \cup \mathrm{Cl}\left(F_{2}\left(b_{2}\right)\right)$, where $F_{n}\left(b_{n}\right), n=1,2$, are any choice of rays. This is possible by Proposition 3.4.

Let integers $u$ and $v$, and particular rays $F_{1}\left(b_{1}\right)$ and $F_{2}\left(b_{2}\right)$ be chosen, so that

(1) $T^{u} p$ and $T^{v} p$ are not separated by $\beta \cup \mathrm{Cl}\left(F_{1}\left(b_{1}\right)\right) \cup \mathrm{Cl}\left(F_{2}\left(b_{2}\right)\right)$, and 
(2) $u-v=2 k$, with $k>N$.

This is possible by Proposition 3.5.

Now apply $T^{l}$, where $l=k-u$. We conclude that the points $T^{-k} p$ and $T^{k} p$ are separated by $\beta \cup \mathrm{Cl}\left(F_{1}\left(b_{1}\right)\right) \cup \mathrm{Cl}\left(F_{2}\left(b_{2}\right)\right)$, but are not separated by

$$
T^{l} \beta \cup T^{l}\left(\mathrm{Cl}\left(F_{1}\left(b_{1}\right)\right) \cup \mathrm{Cl}\left(F_{2}\left(b_{2}\right)\right)\right) .
$$

We now claim that $T^{ \pm k} p$ are separated by $K\left(T^{l} \beta, \beta\right)$. For suppose not. The set $C=T^{l} \beta \cup T^{l}\left(\mathrm{Cl}\left(F_{1}\left(b_{1}\right)\right) \cup \mathrm{Cl}\left(F_{2}\left(b_{2}\right)\right)\right)$ is of the type treated in Proposition 3.3; thus its intersection with $K\left(T^{l} \beta, \beta\right)$ is connected.

Hence, from App. 2, we would have $T^{ \pm k} p$ not separated by $C \cup K\left(T^{l} \beta, \beta\right)$. But this set contains $\beta \cup \mathrm{Cl}\left(F_{1}\left(b_{1}\right)\right) \cup \mathrm{Cl}\left(F_{2}\left(b_{2}\right)\right)$, and we know that $T^{ \pm k} p$ are separated by this set.

Hence $T^{-k} p$ and $T^{k} p$ lie in different components of $R^{2}-K\left(T^{l} \beta, \beta\right)$. Since $K\left(T^{l} \beta, \beta\right)$ is compact, one of these components is bounded. After an application of $T^{k}$ or $T^{-k}$, one sees that $p$ itself is in a bounded component of $K\left(T^{r} p, T^{s} p\right)$ for certain $r, s$. Q.E.D.

4. Definition. An open set $\Omega \subset \boldsymbol{R}^{2}$ is simply connected if, given any Jordan curve $\Gamma \subset \Omega$, it is true that int $\Gamma \subset \Omega$.

Proposition 4.1. Let $C \subset \boldsymbol{R}^{2}$ be compact, and suppose that $p \in \boldsymbol{R}^{2}$ is in a bounded component of $\boldsymbol{R}^{2}-C$. Let $\Omega \subset \boldsymbol{R}^{2}$ be a simply connected open set.

If $C \subset \Omega$ then $p \in \Omega$.

Proof. Let $\mathcal{O}$ be the component of $\boldsymbol{R}^{2}-C$ which contains $p$. Then $\overline{\mathcal{O}}$ is a connected compact set whose boundary lies in $\Omega$.

Let $\varepsilon>0$ be arbitrary. By App. 3, there exists a Jordan curve $\Gamma$ such that $\overline{\mathcal{O}} \subset$ int $\Gamma$, and each point of $\Gamma$ is within $\varepsilon$ of a point of $\overline{\mathcal{O}}$. Since the boundary of $\overline{\mathcal{O}}$ is in the open set $\Omega$, we conclude that $\Gamma \subset \Omega$ if $\varepsilon$ is small enough.

By assumption $\Omega$ is simply connected; therefore int $\Gamma \subset \Omega$. Since $p \in \mathcal{O} \subset$ int $\Gamma$, we are done. Q.E.D.

Definition. Let $\Omega \subset \boldsymbol{R}^{2}$ be an open set and $T: \boldsymbol{R}^{2} \rightarrow \boldsymbol{R}^{2}$ a free mapping. Then $\Omega$ is a regular domain for $T$ if $\Omega$ is connected, simply connected, and satisfies $T \Omega=\Omega$.

THEOREM 4. Let $F_{1}$ and $F_{2}$ be flow lines with $F_{1} \cap F_{2}=\varnothing$. Let $\alpha$ be a polygonal arc with endpoints $a_{1}$ and $a_{2}$; suppose that $\alpha \cap T \alpha=\varnothing$, and $\alpha \cap F_{n}=\left\{a_{n}\right\}$ for $n=1,2$.

Now let $\Omega \subset \boldsymbol{R}^{2}$ be a regular domain for $T$. If $F_{1} \cup F_{2} \subset \Omega$ then $\alpha \subset \Omega$.

Proof. Because $\Omega$ is connected, there exists an arc $\gamma \subset \Omega$ such that $\gamma$ meets both $F_{1}$ and $F_{2}$.

By Proposition 3.1, the set $\alpha \cup F_{1}$ meets $\mathrm{Cl}\left(F_{2}\right)$ in the single point $a_{2} \in F_{2}$. Hence a subarc $\gamma^{\prime} \subset \gamma$ can be chosen so that $\gamma^{\prime} \cap F_{1} \neq \varnothing$, and $\alpha \cup F_{1} \cup \gamma^{\prime}$ meets $\mathrm{Cl}\left(F_{2}\right)$ in exactly two points $\left\{a_{2}, b_{2}\right\}$, where $b_{2}$ is an endpoint of $\gamma^{\prime}$.

Because $\alpha \cup F_{1} \cup \gamma^{\prime}$ is arcwise connected, we conclude from the results of $\S 1$ that $b_{2} \in F_{2}$. 
Now the set $\alpha \cup F_{2}$ meets $\mathrm{Cl}\left(F_{1}\right)$ in the single point $a_{1} \in F_{1}$. Hence a subarc $\gamma^{\prime \prime} \subset \gamma^{\prime}$ can be chosen so that $b_{2} \in \gamma^{\prime \prime}$, and $\alpha \cup F_{2} \cup \gamma^{\prime \prime}$ meets $\bar{F}_{1}$ in exactly two points $\left\{a_{1}, b_{1}\right\}$ where $b_{1} \in \gamma^{\prime \prime}$.

Again it follows from the results of $\S 1$ that $b_{1}$ is actually in $F_{1}$.

Put $\beta=\gamma^{\prime \prime}$. Then $\beta$ has endpoints $b_{1}$ and $b_{2}$, and satisfies $\beta \subset \Omega$ and $\beta \cap F_{n}=\left\{b_{n}\right\}$ for $n=1$, 2. For a given $p \in \alpha$, Theorem 3 implies that $p \in \operatorname{int} K\left(T^{r} \beta, T^{s} \beta\right)$ for certain $r$, s. But $K\left(T^{r} \beta, T^{s} \beta\right)$ is a compact subset of $\boldsymbol{R}^{2}$, completely contained in $\Omega$.

Hence by Proposition 4.1 we have $p \in \Omega$. Q.E.D.

5. We begin by defining the natural way to proceed from an open set to the simply connected open set it determines.

Definition. Let $\mathcal{O}$ be an arbitrary open subset of $\boldsymbol{R}^{2}$.

Then $S C(\mathcal{O})=\bigcup_{\Gamma \subset \mathcal{O}}$ int $\Gamma$, where $\Gamma$ runs through all Jordan curves contained in $\mathcal{O}$.

Note that $S C(\mathcal{O})$ is open and contains the original set $\mathcal{O}$.

Proposition 5.1. Let $\mathcal{O}$ be open, and let $K$ be a compact connected subset of $S C(\mathcal{O})$. Then there is a Jordan curve $\Gamma \subset \mathcal{O}$ with $K \subset$ int $\Gamma$.

Proof. By the definition of $S C(\mathcal{O})$ and by compactness of $K$, there exist finitely many Jordan curves $\left\{\Gamma_{k}\right\}$, with $K \subset \bigcup$ (int $\Gamma_{k}$ ) and $\Gamma_{k} \subset \mathcal{O}$ for all $k$.

Put $K^{\prime}=\bigcup \mathrm{Cl}$ (int $\Gamma_{k}$ ). Then $K^{\prime}$ is compact, connected, and its boundary $\partial K^{\prime}$ satisfies $\partial K^{\prime} \subset \mathcal{O}$.

Therefore, by App. 3, there exists a Jordan curve $\Gamma$ with $K^{\prime} \subset$ int $\Gamma$ and $\Gamma \subset \mathcal{O}$. Q.E.D.

Proposition 5.2. For any open set $\mathcal{O}$, the set $S C(\mathcal{O})$ is simply connected.

Proof. Let $\Gamma \subset S C(\mathcal{O})$ be any Jordan curve.

Since $\Gamma$ is compact, we can by Proposition 5.1 find a Jordan curve $\Gamma^{\prime} \subset \mathcal{O}$ with $\Gamma \subset$ int $\Gamma^{\prime}$.

Hence int $\Gamma \subset$ int $\Gamma^{\prime}$, where int $\Gamma^{\prime} \subset S C(\mathcal{O})$ by the definition of $S C(\mathcal{O})$. Q.E.D.

THEOREM 5. Let $\Omega$ be a regular domain with $\Omega \neq \boldsymbol{R}^{2}$. Let $p$ be a boundary point of $\Omega$. Let $D_{0}$ be a disc centered at $p$ which satisfies $\mathrm{Cl}\left(D_{0}\right) \cap \mathrm{Cl}\left(T D_{0}\right)=\varnothing$.

Then there exists a regular domain $\Omega^{\prime}$ which satisfies $\Omega \subset \Omega^{\prime}, D_{0} \subset \Omega^{\prime}$, and $\Omega^{\prime} \neq \boldsymbol{R}^{2}$.

Proof. Let $D$ be the largest open disc centered at $p$ which satisfies $D \cap T D=\varnothing$. Let a point $q$ be chosen so that $q$ and $T q$ are both on the boundary of $D$.

Choose simple polygonal arcs $m_{1}$ and $m_{2}$ with the following properties: $m_{1}$ and $m_{2}$ both have endpoints $q, T q ; m_{1} \cup m_{2}$ is a Jordan curve with $\mathrm{Cl}\left(D_{0}\right)$ in its interior; and $m_{1} \cup m_{2}-\{q, T q\} \subset D$.

Put $\mathcal{O}=$ int $\left(m_{1} \cup m_{2}\right)$; we have $D_{0} \subset \mathcal{O}$. Set $\Omega^{\prime}=S C\left(\cup \pm_{\infty}^{\infty} T^{n} \mathcal{O} \cup \Omega\right)$. We claim that $\Omega^{\prime}$ satisfies all the conditions of Theorem 5 .

It is trivial to verify that $\Omega^{\prime}$ is a regular domain; connectivity follows from $\mathcal{O} \cap \Omega \neq \varnothing$, and simple connectivity follows from Proposition 5.2. 
The only difficult thing to verify is that $\Omega^{\prime} \neq \boldsymbol{R}^{2}$, and this indeed is the heart of the matter.

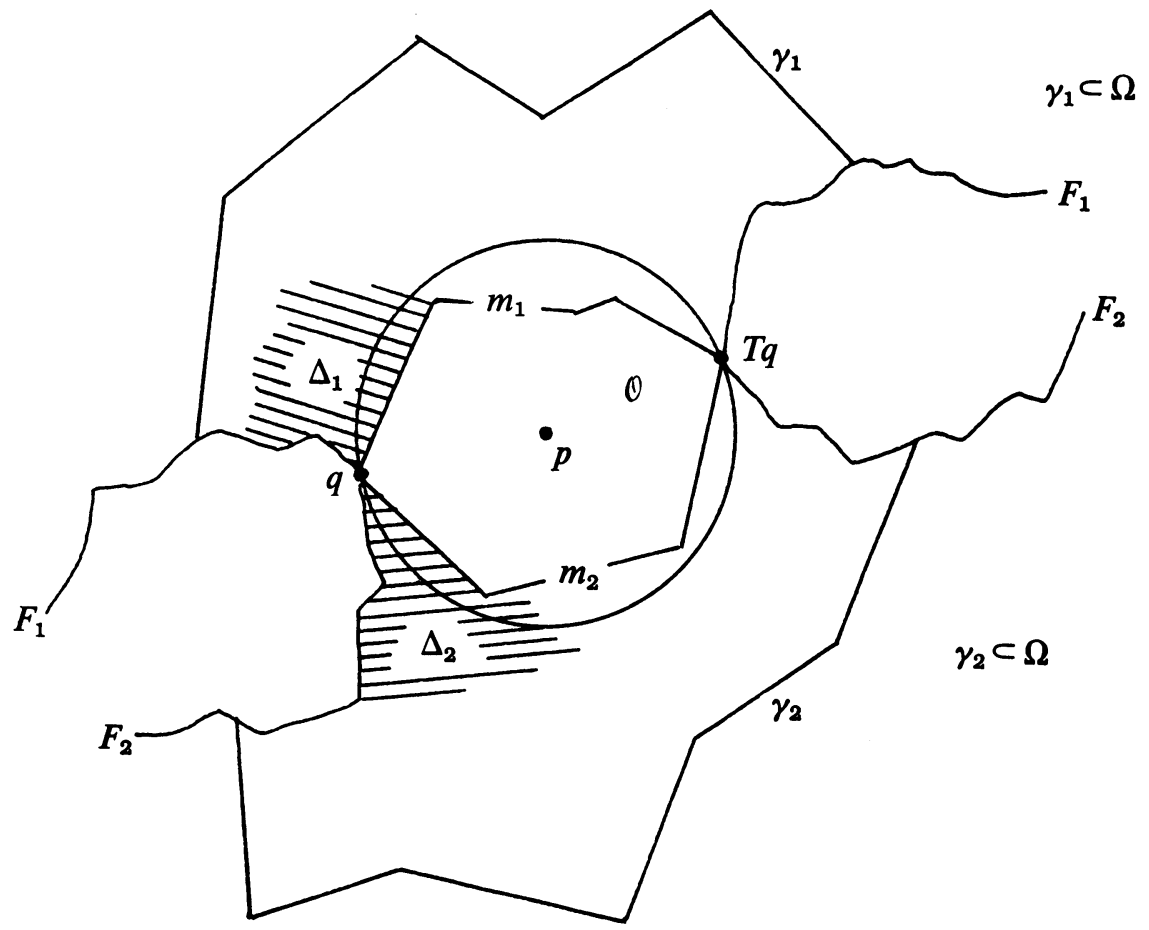

Put $F_{k}=\bigcup_{n} T^{n} m_{k}$ for $k=1,2$; then the $F_{k}$ are flow lines by virtue of the construction of $m_{k}$. Let $\Delta_{1}$ be the side domain of $F_{1}$ which avoids $m_{2}$; likewise let $\Delta_{2}$ be that side domain of $F_{2}$ which avoids $m_{1}$.

Then the sets $\Delta_{1}, \Delta_{2}$, and $\bigcup_{n} T^{n} \mathcal{O}$ are mutually disjoint.

Now suppose, if possible, that $\Omega^{\prime}=S C\left(\cup_{n} T^{n} \mathcal{O} \cup \Omega\right)$ is all of $\boldsymbol{R}^{n}$. By Proposition 5.1 there exists a polygonal Jordan curve $\Gamma \subset \bigcup_{n} T^{n} \mathcal{O} \cup \Omega$ which satisfies $m_{1} \cup m_{2}$ $\subset$ int $\Gamma$.

We apply Theorem 2.5 to the flow lines $F_{k}$ and the side domains $\Delta_{k}$. The conclusion is that $\Gamma$ contains closed subarcs $\gamma_{1}$ and $\gamma_{2}$ with the following properties:

(1) $\gamma_{k}$ is, except for its endpoints, contained in $\Delta_{k}$, and

(2) $\gamma_{k}$ meets both components of $F_{k}-m_{k}$.

Now $\gamma_{k} \subset \bigcup_{n} T^{n} \mathcal{O} \cup \Omega$; since $\gamma_{k}$ is contained in $\mathrm{Cl}\left(\Delta_{k}\right)$ which avoids $\cup_{n} T^{n} \mathcal{O}$, we must conclude that $\gamma_{k} \subset \Omega$ for $k=1,2$.

Because $\gamma_{k}$ meets both components of $F_{k}-m_{k}$ while it avoids $m_{k}$, we have by Proposition 1.9 that $\gamma_{k} \cap T \gamma_{k} \neq \varnothing$ for $k=1,2$.

We now claim that for each side domain $\Delta_{k}$ there exists a flow line $F_{k}^{\prime}$ and a polygonal arc $\beta_{k}$ which have the following properties:

(1) $\beta_{k} \cap F_{k}^{\prime}=\left\{b_{k}^{\prime}\right\}$ and $\beta_{k} \cap F_{k}=\left\{b_{k}\right\}$ where $b_{k}, b_{k}^{\prime}$ are the endpoints of $\beta_{k}$; 
(2) $\beta_{k} \cap T \beta_{k}=\varnothing$; and

(3) $F_{k}^{\prime} \subset \Omega \cap \Delta_{k}$.

The construction of $F_{k}^{\prime}$ and $\beta_{k}$ is done as follows. Let $b_{k}$ be one endpoint of $\gamma_{k}$. Let $\gamma_{k}^{\prime} \subset \gamma_{k}$ be a subarc with endpoints $b_{k}$ and $c_{k}$; let it be the smallest such subarc which satisfies $\gamma_{k}^{\prime} \cap T\left(\gamma_{k}^{\prime}\right) \neq \varnothing$. Then $\gamma_{k}^{\prime}$ contains $T c_{k}$ or $T^{-1} c_{k}$; let $b_{k}^{\prime}$ denote this point.

Now $\gamma_{k}^{\prime}$ is the union of two arcs; $\beta_{k}$ from $b_{k}$ to $b_{k}^{\prime}$, and $\delta_{k}$ from $b_{k}^{\prime}$ to $c_{k}$. Put $F_{k}^{\prime}=\bigcup T^{n} \delta_{k}$; then the flow line $F_{k}^{\prime}$ and the $\operatorname{arc} \beta_{k}$ satisfy all the stipulations (1)-(3).

Replacing $\beta_{k}$ by one of its iterates $T^{n} \beta_{k}$, we can assume that $b_{k} \in m_{k}$.

Now choose a polygonal arc $\tau$, with endpoints $b_{1}$ and $b_{2}$, which contains $p$ and satisfies $\tau-\left\{b_{1}, b_{2}\right\} \subset$ int $\left(m_{1} \cup m_{2}\right)$. Finally consider $\sigma=\beta_{1} \cup \tau \cup \beta_{2}$.

The three constituents of $\sigma$ are respectively contained in the three disjoint invariant sets $\Delta_{1}, \cup T^{n} \mathcal{O}$, and $\Delta_{2}$. Moreover, the three constituents are separately disjoint from their images; therefore $\sigma$ itself satisfies $\sigma \cap T \sigma=\varnothing$.

To summarize: The original point $p \in \partial \Omega$ is now contained in a polygonal arc $\sigma$, which meets the two flow lines $F_{1}^{\prime}$ and $F_{2}^{\prime}$ only in its endpoints. Moreover $F_{1}^{\prime}$ and $F_{2}^{\prime} \subset \Omega$.

Hence, we conclude by Theorem 4 that $p \in \Omega$. But this contradicts the fact that $p$ was chosen to be in the boundary of $\Omega$. Q.E.D.

6. The announced theorems in the Introduction are deduced in the following fashion.

THEOREM 6.1. Let $A \subset \boldsymbol{R}^{2}$ be a compact set, and let $\Omega_{0} \subset \boldsymbol{R}^{2}$ be a regular domain for $T$, such that $\Omega_{0} \neq R^{2}$.

Then there exists a regular domain $\Omega$, such that $A \cup \Omega_{0} \subset \Omega$ and $\Omega \neq \boldsymbol{R}^{2}$.

Proof. Replacing $A$ by a larger compact set if necessary, we can assume that $A \cap \Omega_{0} \neq \varnothing$.

Choose $\varepsilon>0$ so that $\mathrm{Cl}\left(D_{\varepsilon}(a)\right) \cap \mathrm{Cl}\left(T\left(D_{\varepsilon}(a)\right)\right)=\varnothing$ for all $a \in A .\left(D_{\varepsilon}(a)\right.$ is the open disc of radius $\varepsilon$ centered at $a$.)

Choose $N>0$ so that for any set of points $a_{1}, \ldots, a_{N}$ of $A$ we have $d\left(a_{i}, a_{j}\right)<\varepsilon$ for some $i \neq j$.

If $\Omega_{0} \ngtr A$, choose $a_{1} \in A \cap \partial \Omega_{0}$ and apply Theorem 5 to $D_{\varepsilon}\left(a_{1}\right)$. The result is a regular domain $\Omega_{1} \neq \boldsymbol{R}^{2}$ with $\Omega_{1} \supset D_{\varepsilon}\left(a_{1}\right) \cup \Omega_{0}$.

If $\Omega_{1} \ngtr A$, choose $a_{2} \in A \cap \partial \Omega_{1}$ and repeat the procedure, obtaining a regular domain $\Omega_{2} \neq R^{2}$ with $\Omega_{2} \supset D_{\varepsilon}\left(a_{2}\right) \cup \Omega_{1} \supset D_{\varepsilon}\left(a_{2}\right) \cup D_{\varepsilon}\left(a_{1}\right) \cup \Omega_{0}$.

Since $a_{k} \in \partial \Omega_{k-1}$ and $\Omega_{k-1} \supset D_{\varepsilon}\left(a_{k-1}\right) \cup \cdots \cup D_{\varepsilon}\left(a_{1}\right)$ we have that the distance from $a_{k}$ to $a_{s}$ is $\geqq \varepsilon$ for all $k>s$.

Hence this iterative procedure must exhaust $A$ in at most $N$ steps. The result is a regular domain $\Omega_{m}$ with $\Omega_{m} \neq \boldsymbol{R}^{2}$ and $\Omega_{m} \supset \Omega_{0} \cup A$. We then take $\Omega=\Omega_{m}$ in the statement of the theorem. Q.E.D.

THEOREM 6.2. Let $T: R^{2} \rightarrow R^{2}$ be a free mapping. 
Then for any compact set $A \subset R^{2}$ there is an unbounded connected set $B \subset R^{2}$ which avoids $\cup T^{n} A$.

Proof. Let $\Omega_{0}$ be any regular domain with $\Omega_{0} \neq \boldsymbol{R}^{2}$.

For example, one could take $\Omega_{0}$ to be a side domain of a flow line.

By Theorem 6.1 there is a regular domain $\Omega_{1}$ with $A \subset \Omega_{1}$ and $\Omega_{1} \neq R^{2}$. By App. 7, $\boldsymbol{R}^{2}-\Omega_{1}$ contains an unbounded connected set. Q.E.D.

THEOREM 6.3. Let $E \subset \boldsymbol{R}^{2}$ be the closed upper half-plane.

Let $T$ be a homeomorphism of $E$ onto itself which has no fixed points and preserves orientation.

Then for any compact set $A \subset E$ there is an unbounded connected set $B \subset E$ which avoids $\cup T^{n} A$.

Proof. Let $J: \boldsymbol{R}^{2} \rightarrow \boldsymbol{R}^{2}$ be the reflection $(x, y) \leftrightarrow(x,-y)$. Extend $T$ to be a mapping $T^{\prime}$ of the whole plane by setting $T^{\prime}=J T J$ on the lower half-plane. The $T^{\prime}$ is easily seen to preserve orientation and have no fixed points; by Proposition 0 it is a free mapping of $\boldsymbol{R}^{2}$ onto itself.

Let $\Omega_{0}$ be the open lower half-plane; it is a regular domain for $T^{\prime}$. By Theorem 6.1 there is an open set $\Omega_{1} \subset R^{2}$ which is a regular domain for $T^{\prime}$ and satisfies $A \cup \Omega_{0} \subset \Omega_{1}$ and $\Omega_{1} \neq \boldsymbol{R}^{2}$. Thus, we conclude from App. 7 that $\boldsymbol{R}^{2}-\Omega_{1}$ contains an unbounded connected set $C$; by construction $C \subset E$. Q.E.D.

Appendix. App. 1 (Jordan Curve Theorem). Let $\Gamma \subset \boldsymbol{R}^{2}$ be a Jordan curve. Then $\boldsymbol{R}^{2}-\Gamma$ has two components $D_{1}$ and $D_{2}$; moreover $\Gamma \subset \mathrm{Cl}\left(D_{n}\right)$ for $n=1,2$.

APP. 2 (AleXANDeR's Lemma). Let $C \subset \boldsymbol{R}^{2}$ be closed and $K \subset \boldsymbol{R}^{2}$ be compact, and let $p, q$ be two points of $\boldsymbol{R}^{2}-(C \cup K)$.

Suppose that $p$ and $q$ are not separated by $C$ and not separated by $K$. If $C \cap K$ is connected then $p$ and $q$ are not separated by $C \cup K$.

For proofs of Apps. 1 and 2 see Newman [2, pp. 101-116].

Recall that $D_{\varepsilon}(p)$ is the open disc of radius $\varepsilon>0$ centered at the point $p \in \boldsymbol{R}^{2}$.

APP. 3. Let $K$ be a compact connected subset of $\boldsymbol{R}^{2}$, and let $\varepsilon>0$ be arbitrary. Then there exists a (polygonal) Jordan curve $\Gamma$ with $\Gamma \cap K=\varnothing . K \subset$ int $\Gamma$, and such that $D_{\varepsilon}(p) \cap K \neq \varnothing$ for all $p \in \Gamma$.

The remaining facts in this Appendix can be deduced from Apps. 1, 2, and 3.

APP. 4. Let $\Gamma \subset R^{2}$ be a Jordan curve, and let $K_{1}, K_{2} \subset R^{2}$ be disjoint compact connected sets which satisfy $K_{n} \subset \mathrm{Cl}$ (int $\Gamma$ ) and $K_{n} \cap \Gamma=\gamma_{n}$ for $n=1,2$, where $\gamma_{1}$ and $\gamma_{2}$ are disjoint closed subarcs of $\Gamma$.

Then there exists an arc $\alpha$, with endpoints $a_{1}$ and $a_{2}$, such that

(1) $\alpha \subset \mathrm{Cl}$ (int $\Gamma$ ) and $\alpha \cap \Gamma=\left\{a_{1}, a_{2}\right\}$,

(2) $\alpha \cap\left(K_{1} \cup K_{2}\right)=\varnothing$, and

(3) $a_{1}$ and $a_{2}$ are in the two components of $\Gamma-\left(\gamma_{1} \cup \gamma_{2}\right)$.

APP. 5. Let $\Gamma \subset \boldsymbol{R}^{2}$ be a Jordan curve, and let $K \subset \boldsymbol{R}^{2}$ be a compact connected set which satisfies $K \subset \mathrm{Cl}$ (int $\Gamma$ ) and $K \cap \Gamma=\gamma$, when $\gamma \subset \Gamma$ is a subarc with endpoints $b_{1}$ and $b_{2}$. Let $\varepsilon>0$ be arbitrary. 
Then there exists an arc $\alpha$, with endpoints $a_{1}$ and $a_{2}$, such that

(1) $\alpha \subset \mathrm{Cl}$ (int $\Gamma$ ) and $\alpha \cap \Gamma=\left\{a_{1}, a_{2}\right\}$,

(2) $\alpha \cap K=\varnothing$ but $D_{\varepsilon}(p) \cap K \neq \varnothing$ for all $p \in \alpha$,

(3) $D_{\varepsilon}\left(a_{n}\right)$ contains $b_{n}$ for $n=1,2$.

APP. 6. Let $\Gamma_{1}$ and $\Gamma_{2}$ be Jordan curves, and suppose that $\Gamma_{1} \cap \Gamma_{2}$ is a common subarc. If int $\Gamma_{1} \cap$ int $\Gamma_{2} \neq \varnothing$ then either int $\Gamma_{1} \subset$ int $\Gamma_{2}$ or int $\Gamma_{2} \subset$ int $\Gamma_{1}$.

APP. 7. Let $\Omega$ be a simply connected open subset of $\boldsymbol{R}^{2}$. If $\Omega \neq \boldsymbol{R}^{2}$ then there exists an unbounded closed connected set $C$ which avoids $\Omega$.

A proof of App. 7 is outlined as follows. Choose a fixed $p_{0} \notin \Omega$, and say that a closed set $E$ is "good" if $p_{0} \in E, E \cap \Omega=\varnothing$, and $E \cap \Gamma \neq \varnothing$ for every Jordan curve $\Gamma$ which contains $p_{0}$ in its interior domain. For example, $\boldsymbol{R}^{2}-\Omega$ is good.

The collection of good sets is invariant under arbitrary nested intersections. By Zorn's lemma, take $C$ to be a minimal good set. This $C$ satisfies all the conditions of App. 7; for example, the connectivity of $C$ follows from App. 3.

\section{REFERENCES}

1. L. E. J. Brouwer, Beweis des ebenen Translationssatzes, Math. Ann. 72 (1912), 37-54.

2. M. H. A. Newman, Elements of the topology of plane sets of points, Cambridge Univ. Press, New York, 1961. MR 24 \#A2374.

3. S. A. Andrea, On homeomorphisms of the plane, and their embedding in flows, Bull. Amer. Math. Soc. 71 (1965), 381-383. MR 30 \#2478.

4. - On homeomorphisms of the plane which have no fixed points, Abh. Math. Sem. Univ. Hamburg 30 (1967), 61-74. MR 34 \#8397.

University of California at San Diego, La Jolla, California 92037 